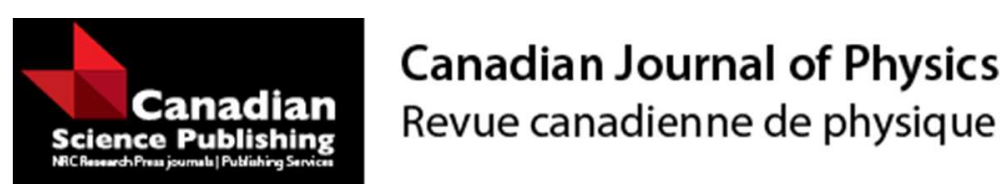

\title{
Optical Characteristics of Finite Temperature Quantum Electron Gas
}

\begin{tabular}{|r|l|}
\hline Journal: & Canadian Journal of Physics \\
\hline Manuscript ID & cjp-2017-0182.R1 \\
\hline Manuscript Type: & Article \\
\hline Date Submitted by the Author: & 05-May-2017 \\
\hline Complete List of Authors: & Akbari-Moghanjoughi, M.; Azarbaijan Univ Tarbiat Moallem, \\
\hline Keyword: & $\begin{array}{l}\text { Electron Gas, Quantum Plasmas, Optical Properties, Dielectric constant, } \\
\text { Refraction Coefficient }\end{array}$ \\
\hline $\begin{array}{r}\text { Is the invited manuscript for } \\
\text { consideration in a Special } \\
\text { Issue? : }\end{array}$ & N/A \\
\hline
\end{tabular}




\title{
Optical Characteristics of Finite Temperature Quantum Electron Gas
}

\author{
M. Akbari-Moghanjoughi ${ }^{1}$ \\ ${ }^{1}$ Faculty of Sciences, Department of Physics, \\ Azarbaijan Shahid Madani University, 51745-406 Tabriz, Iran
}

\begin{abstract}
In this paper we study the propagation of elliptically polarized transverse electromagnetic waves in a quasineutral plasma with arbitrary degenerate electron fluid ranging from classical dilute to fully degenerate density regimes by using the appropriate collisional magnetohydrodynamics model which incorporates the adiabatic quantum equation of states $(\mathrm{EoS})$ for high frequency electron fluid compression and the Bohm quantum potential responsible for the collective quantum diffraction effect. Three distinct propagation modes are categorized corresponding to electron density regimes of dilute, intermediate and fully degenerate plasmas. One of these modes is found to be purely of quantum mechanical origin and disappears in the absence of the quantum diffraction effect. The present generalized magnetohydrodynamics theory qualitatively describes key features of optical parameters and experimental data of dilute classical, semiconducting and in fully degenerate plasmas. Different optical parameters such as the refractive and absorption index of the new mode are investigated. It is shown that the optical response of the magnetized plasma with arbitrary degeneracy is essentially governed by three characteristic frequencies namely that of collision, plasmon and cyclotron ones. The profound experimental minimum in the refractive index of arbitrary degenerate quantum plasmas and its dependence on the characteristic frequencies is studied in detail. Current investigation is of fundamental importance in high energy density and fusion plasma diagnostics and may provide key knowledge on the characteristics of astrophysical dense matter.
\end{abstract}

PACS numbers: 52.30.-q,71.10.Ca, 05.30.-d 


\section{INTRODUCTION}

The electromagnetic response of electron fluid is one of the fundamental diagnostic tools in laboratory and astrophysics plasmas. For instance, much information can be deduced from optical spectrum of exotic states of matter like high energy density (HED) [1], relativistic degenerate [2-4] and warm dense matter (WDM) [5]. However, full understanding of the exact physical mechanisms involved in the optical features of matter requires a perfect knowledge about collective behavior of electrons which has been the subject of intense modern physics research for past few decades [6-11]. One of the elementary collective aspects of the free electron fluid is the plasma oscillations $[12,13]$ and the resonance condition which is well described by the most basic Drude model [14, 15]. However, to capture the essential physics of collective behavior of the free electron fluid beyond that of the classical models say in compressed metals, one needs to consider the appropriate statistical and thermodynamic features such as the equation of state $(\mathrm{EoS})$ and the quantum electron diffraction effect which become dominant when the electron number density increases. This is due to the fact that the inter-electron distances compare to the de Broglie thermal wavelength in which case the electron fluid starts to behave quantum mechanically [16-19]. However, in order to fully understand the structure of optical response in free electron systems like metals one should take into account the effects due to bound electrons as well such as the screening of conduction electrons by s and d electrons which lead to hybrid resonance in silver, for example [20, 21]. This however requires a tedious task of band structure calculation [22].

On the other hand, quantum magnetohydrodynamic (QMHD) models [23, 24] are frameworks incorporating many essential features of the physical model under consideration to provide effective description of basic features of the system at the lowest cost. They are perfect extensions to Drude-like models which fail to describe the quantum mechanical nature of the many-body systems. Moreover, the use of more powerful quantum kinetic transport theories are very limited to the simplified cases due to the mathematical complexity. Also, the time consuming large scale Vlasov-Poisson and Wigner-Poisson simulation methods, although may provide a detailed information on the behavior of the system, they fail to bring into view a transparent physical insight into the problem and are more pron to computational artifacts. Very early application of QHD theory on two stream instability has revealed that unlike classical ones quantum plasmas possess no threshold [25]. Recently, the 
Bohm potential effect on plasmon spectrum of graphene has been studied using QHD theory [26]. Quantum hydrodynamic (QHD) and QMHD have been employed to study properties of superdense astrophysical plasmas [27, 28], nano-particles [29, 30] and semiconductors [31-34]. Some new interesting features of quantum electron fluid has been predicted using hydrodynamic calculation in recent years [35-39]. For instance study of different instability mechanisms in quantum plasmas reveals features unseen in ordinary classical plasmas $[40,41]$. Application of QHD theory to charge screening in plasmas shows the existence of attractive force among ions of same charge caused by fully [42] or partially [43] degenerate electron fluid. Numerous interesting features of linear and nonlinear waves in dense plasmas have been reported during the past few years [44-49]. In this paper by using the appropriate QMHD model we study the dispersion relation for elliptically polarized transverse electromagnetic waves and show that the refraction index of electron fluid with arbitrary degeneracy predict the essential features of free electron fluid in metals. Many other interesting properties of the free electron gas is discussed in terms of different parameters such as the electron temperature and density, magnetic field strength and collision frequency. It is shown that three fundamental frequencies, namely, plasmon, cyclotron and collision rule many of the optical characteristics of the electron fluid from classical up to fully degenerate quantum density regime. The paper is organized in the following manner. We provide the appropriate QMHD model in Sec II and derive the dielectric function and optical parameters in Sec. III. A numerical discussion is given in Sec. IV and finally conclusions are made in Sec. V.

\section{THE MAGNETOHYDRODYNAMIC MODEL}

We consider the dynamics of electron fluid in a neutralizing charged medium subject to a uniform magnetic field. We also take into account the generalized quantum statistical effects on the electron fluid such as the scalar pressure and quantum potential leading to the electron diffraction effect. From the magnetohydrodynamic momentum balance equation we have [50]

$$
\left(\frac{d}{d t}+\nu_{e i}\right) \mathbf{J}-\frac{e^{2} n_{0}}{m_{e}} \mathbf{E}-\frac{e}{m_{e} c} \mathbf{J} \times \mathbf{B}_{\mathbf{0}}-\frac{e n_{0}}{n m_{e}} \nabla P_{G}+\frac{e n_{0} \hbar^{2} \nabla}{2 m_{e}^{2}}\left(\frac{\Delta \sqrt{n}}{\sqrt{n}}\right)=0,
$$


where $d / d t=\partial / \partial t+\left(1 / e n_{0}\right) \mathbf{J} \cdot \nabla$ with $\mathbf{E}, \mathbf{B}_{\mathbf{0}}$ and $\mathbf{J}=e n_{0} \mathbf{V}$ representing the electric field, the constant magnetic field and current density vectors in the fluid. Also, e denotes the electron charge value, $m_{e}$ its mass, $n_{0}$ the equilibrium value of the number-density $n, c$ the speed of light in vacuum and $\hbar$ the scaled Planck's constant. Moreover, the electron fluid is assumed to follow a generalized adiabatic equation of state (EoS) with arbitrary degeneracy leading to the generalized statistical pressure $P_{G}$ as described below [38]

$$
P_{G}=m_{e} n_{0} G_{e} V_{T e}^{2}\left(\frac{n}{n_{0}}\right)^{3}, \quad n=-N_{e} \operatorname{Li}_{3 / 2}\left[-\exp \left(\beta \mu_{e 0}\right)\right],
$$

where $\mu_{e 0}$ is the equilibrium chemical potential of electron fluid, $V_{T e}=\sqrt{1 / \beta m_{e}}$ is the electron thermal speed with $\beta=1 / k_{B} T_{e}$ and $N_{e}=m_{e}{ }^{3 / 2} /\left(\sqrt{2} \hbar^{3} \pi^{3 / 2} \beta^{3 / 2}\right)$. Note that the exponent 3 in the EoS (2) reminds one of the polytropic index $\gamma$ of a classical gas with adiabatic fast compression of electron fluid in high frequency phenomenon like Langmuir oscillations. The parameter $G_{e}$ is the kinetic correction factor in the EoS given as

$$
G_{e}=\frac{\operatorname{Li}_{5 / 2}\left[-\exp \left(\beta \mu_{e 0}\right)\right]}{\operatorname{Li}_{3 / 2}\left[-\exp \left(\beta \mu_{e 0}\right)\right]}
$$

The functions $\mathrm{Li}_{s}$ is the functional representation of the Fermi integrals of order $s$ given as

$$
\operatorname{Li}_{s}\left[-\exp \left(\beta \mu_{e 0}\right)\right]=\frac{-1}{\Gamma(s+1)} \int_{0}^{\infty} \frac{z^{s} \mathrm{~d} z}{\exp \left(z-\beta \mu_{e 0}\right)+1},
$$

where, $\Gamma$ is the standard Gamma-function. Note that the generalized pressure $P_{G}$ represents the scalar pressure for electron fluid of arbitrary degeneracy with the factor $G_{e}$ reducing to values of $\{1,3 / 5\}$ in the extreme dilute classical $\left(\beta \mu_{e 0} \rightarrow-\infty\right)$ and fully degenerate $\left(\beta \mu_{e 0} \rightarrow\right.$ $+\infty)$ electron fluid limits, respectively. The Poisson's equation on the other hand couples the electric field to the fluid density by $\nabla \cdot \mathbf{E}=4 \pi e\left(n_{0}-n\right)$. Finally, the momentum and Poisson's equations are coupled with the Ampere and Faraday laws $\nabla \times \mathbf{B}=4 \pi \mathbf{J} / c+(1 / c) \partial \mathbf{E} / \partial t$ and $\nabla \times \mathbf{E}=-(1 / c) \partial \mathbf{B} / \partial t$ in order to close the magnetohydrodynamic system. In the next section we will use four equations, namely, the electron momentum (with the appropriate generalized EoS defined earlier), the Poisson's, Ampere's and Faraday's which make a complete set in order to eliminate $n, J, E, B$ plasma parameters leading to the desired dispersion relation for elliptically polarized electromagnetic waves [51] in plasmas with arbitrary degree of degeneracy for electrons and the neutralizing ion fluid. 


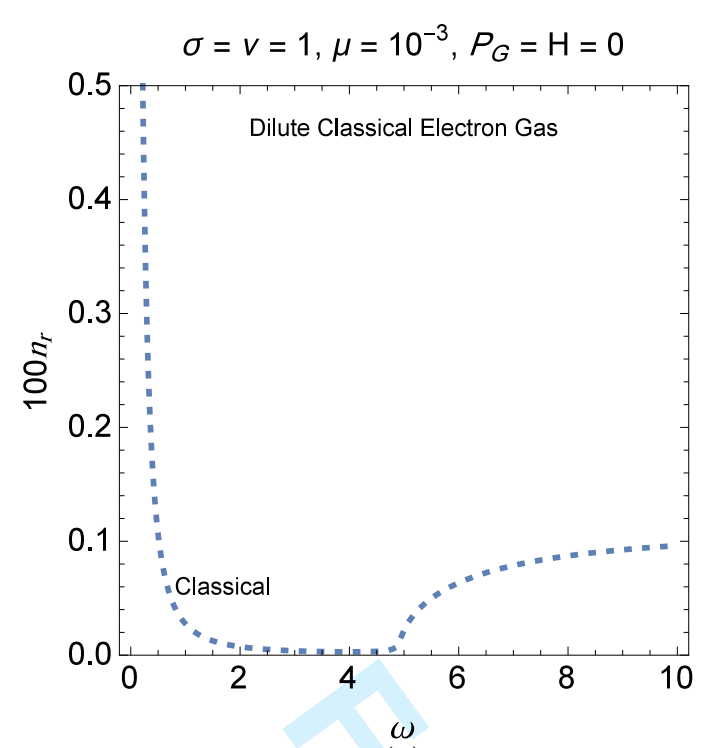

(a)

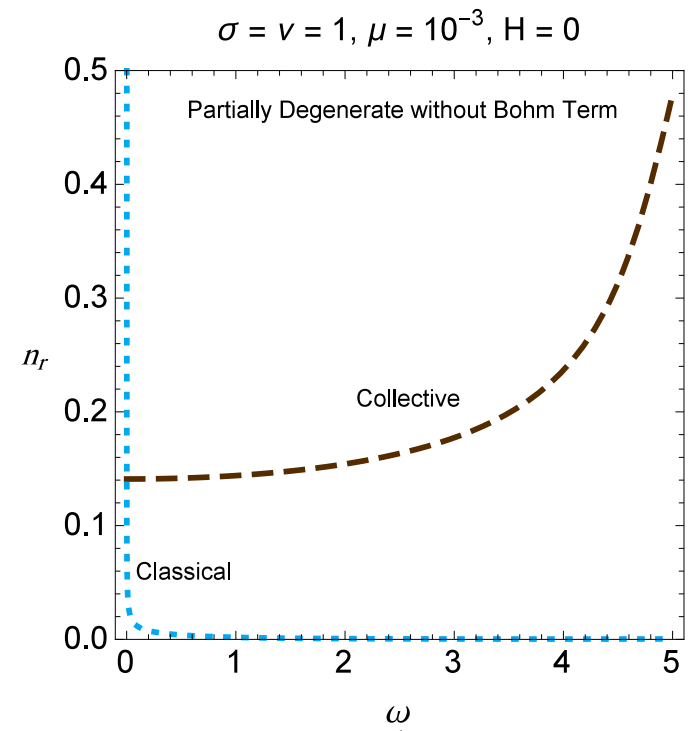

(b)

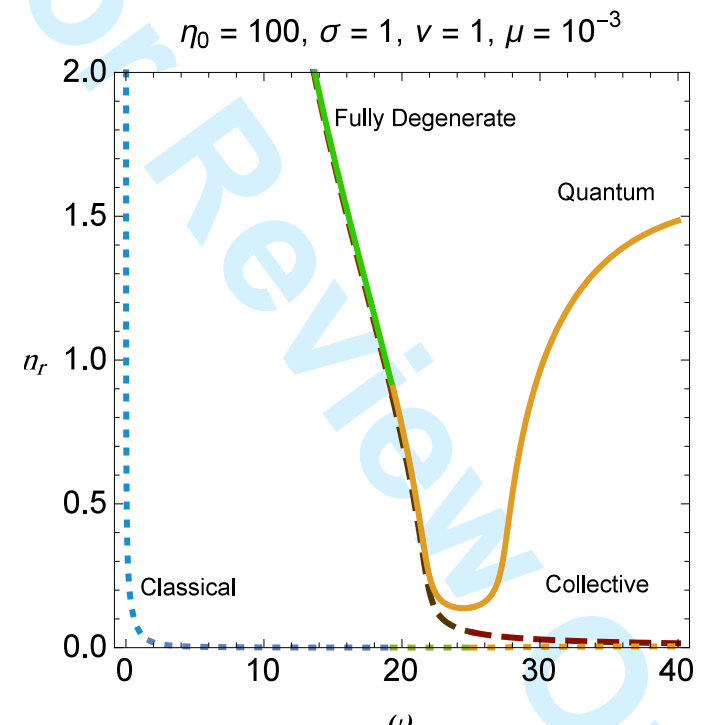

$(c)$

FIG. 1: Three different modes of propagation of eliptically polarized transverse electromagnetic waves with different origin, namely, purely classical (dotted curves), collective (dashed curves), and purely quantum mechanical (solid curve) modes. The latter is due to the collective quantum diffraction of electron fluid arising from the Bohm potential.

\section{DIELECTRIC RESPONSE AND OPTICAL PARAMETERS}

In this section we calculate the dielectric response for linear electromagnetic wave propagation in an arbitrary degenerate quasineutral electron fluid. To this end we set a specific geometry of propagation with the wavenumber in $x$-direction $\mathbf{k}=\{\mathrm{k}, 0,0\}$ and the constant 
magnetic field lying in the $z$-direction $\mathbf{B}_{\mathbf{0}}=\left\{0,0, \mathbf{B}_{0}\right\}$. Further setting the electric field and current density vectors to be in the $x-y$ plane (for the general elliptically polarized electromagnetic extraordinary waves) and assuming linear perturbations of the form

$$
\left[\begin{array}{c}
\mathbf{E} \\
\mathbf{B} \\
\mathbf{J} \\
n
\end{array}\right]=\left[\begin{array}{c}
\mathbf{0} \\
\mathbf{0} \\
\mathbf{0} \\
n_{0}
\end{array}\right]+\left[\begin{array}{c}
\mathbf{E}_{1} \\
\mathbf{B}_{1} \\
\mathbf{J}_{1} \\
n_{1}
\end{array}\right] \exp (i k x-i \omega t)
$$

the Fourier analysis of momentum balance equation (1) leads to

$$
\begin{aligned}
& 4 \pi\left(i \omega-\nu_{e i}\right) \mathrm{J}_{1 x}+\omega_{p e}^{2} \mathrm{E}_{1 x}+4 \pi \omega_{c e} \mathrm{~J}_{1 x}+\frac{m \omega_{p e}^{2}}{e}\left(3 G_{e} V_{T e}^{2}+\frac{\hbar^{2} k^{2}}{4 m^{2}}\right) i k n_{1}=0, \\
& 4 \pi\left(i \omega-\nu_{e i}\right) \mathrm{J}_{1 y}+\omega_{p e}^{2} \mathrm{E}_{1 y}-4 \pi \omega_{c e} \mathrm{~J}_{1 y}=0
\end{aligned}
$$

where $\omega_{c e}=e B_{0} / m_{e} c$ and $\omega_{p e}=\sqrt{4 \pi e^{2} n_{0} / m_{e}}$ are the electron cyclotron and plasmon frequencies, respectively. Also, the magnetic field vector $\mathbf{B}$ can be directly eliminated from the Faraday and Ampere's laws giving rise to the following equations

$$
4 \pi i \omega \mathrm{J}_{1 x}=\omega^{2} E_{1 x}, \quad 4 \pi i \omega \mathrm{J}_{1 y}=\left(\omega^{2}-c^{2} k^{2}\right) E_{1 y}
$$

Moreover, the Poisson's equation linearizes to give $\omega_{p e}^{2} m_{e} n_{1} / e=-i k E_{1 x}$ which then can be used to eliminate the $n_{1}$ from the first equation in (6). Consequently, (6) and (7) can be used to eliminate the current density components which leads to the following secular equation and the dispersion relation for electromagnetic extraordinary waves

$$
\left|\begin{array}{cc}
\frac{\hbar^{2} \omega^{2} N^{4}}{4 m_{e}^{2} c^{4}}+\frac{3 G_{e} V_{T e}^{2} N^{2}}{c^{2}}+\frac{\omega_{p e}^{2}}{\omega^{2}}-\left(1+i \frac{\omega_{p e} \nu_{e i}}{\omega^{2}}\right) & i \frac{\omega_{c e}}{\omega}\left(N^{2}-1\right) \\
i \frac{\omega_{c e}}{\omega} & \frac{\omega_{p e}^{2}}{\omega^{2}}+\left(1+i \frac{\omega_{p e} \nu_{e i}}{\omega^{2}}\right)\left(N^{2}-1\right)
\end{array}\right|=0
$$

where $N=c k / \omega$ is the complex refraction index of the electron fluid with the real and imaginary parts given as $N=n_{r}+i n_{i}$. The equation (8) is a polynomial of degree three in terms of the dielectric constant $\epsilon=N^{2}$ with the real and imaginary components $\epsilon=\epsilon_{r}+i \epsilon_{i}$. The polynomial equation is of the form $A \epsilon^{3}+B \epsilon^{2}+C \epsilon+D=0$ with coefficients given as

$$
\begin{aligned}
& A=\frac{H^{2} \omega^{2}}{4 \alpha^{6}}\left(1+\frac{\mathrm{i} \nu}{\omega}\right), \\
& B=\frac{H^{2} \omega^{2}}{4 \alpha^{2}}\left(\frac{1}{\omega^{2}}-\frac{\mathrm{i} \nu}{\alpha^{2} \omega}-\frac{1}{\alpha^{2}}\right)+\frac{3 \sigma G_{e} V_{T 0}^{2}}{c^{2} \alpha^{4}}\left(1+\frac{\mathrm{i} \nu}{\omega}\right), \\
& C=\mu^{2}+\left(\frac{1}{\omega^{2}}-\frac{\mathrm{i} \nu}{\alpha^{2} \omega}-\frac{1}{\alpha^{2}}\right)\left[\frac{3 \sigma G_{e} V_{T 0}^{2}}{c^{2}}+\left(1+\frac{\mathrm{i} \nu}{\omega}\right)\right], \\
& D=\alpha^{4}\left(\frac{1}{\omega^{2}}-\frac{\mathrm{i} \nu}{\alpha^{2} \omega}-\frac{1}{\alpha^{2}}\right)^{2}-\alpha^{2} \mu^{2} .
\end{aligned}
$$



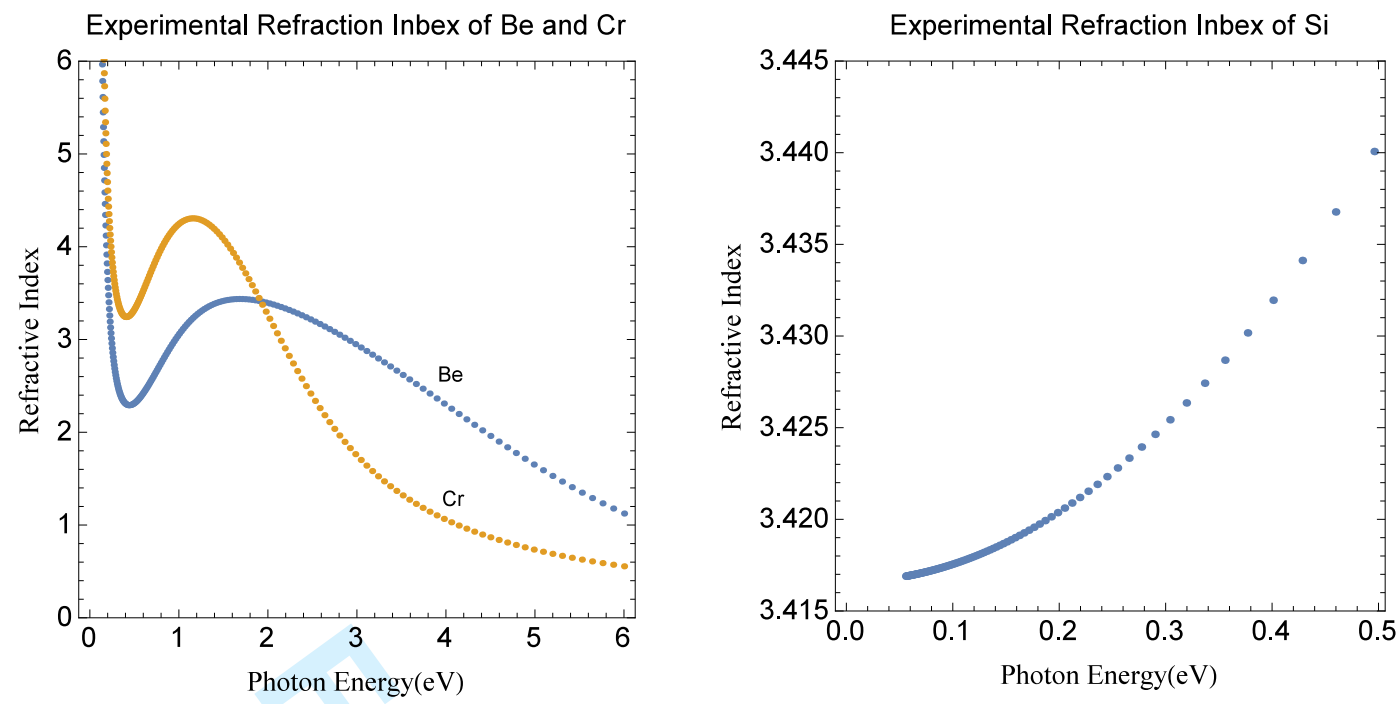

FIG. 2: Experimental refraction index for metallic beryllium and chromium and semiconducting silicon in terms of photon energy (eV) [M. N. Polyanskiy. "Refractive index database," http://refractiveindex.info].

where the scalings $\omega=\omega / \omega_{p 0}$ and $k=k \omega_{p 0} / V_{T 0}$ has been used in which $\omega_{p 0}=\sqrt{4 \pi e^{2} N_{0} / m_{e}}$, $N_{0}=m_{e}^{3 / 2} /\left(\sqrt{2} \hbar^{3} \pi^{3 / 2} \beta_{0}^{3 / 2}\right)$ and $V_{T 0}=\sqrt{k_{B} T_{0} / m_{e}}$ with $T_{0}=300 \mathrm{~K}$ and $\beta_{0}=1 / k_{B} T_{0}$ are the normalizing units. Also, the new parameters are $\mu=\omega_{c e} / \omega_{p 0}, \nu=\nu_{e i} / \omega_{p 0}, H=$ $\hbar \omega_{p 0} / m_{e} c^{2}, \sigma=T_{0} / T_{e}$ and $\alpha=\omega_{p e} / \omega_{p 0}=\sqrt{-\sigma^{3 / 2} \operatorname{Li}_{3 / 2}\left[-\exp \left(\sigma \eta_{0}\right)\right]}$ with $\eta_{0}=\beta_{0} \mu_{e 0}$. It is clearly observed that three characteristic competing frequency parameters $\alpha, \nu$ and $\mu$ control the properties of the refraction index $N$ for the magnetized electron fluid. Furthermore, it is evident from $(9)$ that in the zero-pressure $\left(P_{G}=0\right)$ classical $(H=0)$ electron fluid we have $A=B=0$ and we have one solution (propagation mode) for the characteristic value of the refraction index, $N$. However, for classical $(H=0)$ electron fluid with non-negligible electronic pressure $\left(P_{G} \neq 0\right)$ one obtains two solutions and two propagation modes. On the other hand, for the general case of quantum electron fluid with non-negligible statistical pressure $\left(P_{G} \neq 0\right.$ and $\left.H \neq 0\right)$ one obtains three distinct propagation modes. The remarkable fact is that one of the transverse electromagnetic modes is due purely to the quantum nature of fluid, that is, the quantum diffraction of electrons and Bohm potential. Some new electromagnetic unstable mode with pure quantum origin has also been recently reported in the literature [37]. 


\section{NUMERICAL ANALYSIS AND DISCUSSION}

Figure 1 shows the three different cases of extraordinary electromagnetic wave propagation discussed above. The real part of the refraction index $\left(n_{r}\right)$ is shown for the case of classical electron fluid with negligible statistical pressure effect in Fig. 1(a) for a given set of plasma parameters. Only one mode (dotted curve) is clearly present which we will call it the pure classical mode and it is expected to be observed in the pressureless dilute electron fluid regime. Note that the frequency dependence of this mode is quite similar to that predicted by the basic Drude model [20]. For a classical electron fluid accounting also for the generalized statistical pressure with arbitrary electron degeneracy as shown in Fig. 1(b) a new collective mode appears which is shown as dashed one other than the pure classical mode shown as dotted curve. This mode is expected to be present for density regimes of partially degenerate fluid in semiconductors like silicon and germanium with very low free electron densities where the quantum diffraction effect is negligible. The increase of the refractive index with the photon energy observed in Fig. 1(b) (the dashed curve) is a remarkable property of electron fluid in simple semiconductors [52]. Finally, for the general case of quantum electron gas with both statistical pressure and quantum diffraction effects shown in Fig. 1(c) extra mode appear (solid curve) beside those in Fig. 1(b) which is purely of quantum origin and due to the presence of quantum diffraction of electrons. It is also remarked that the second mode shown with dashed curve in Fig. 1(b) is completely modified in Fig. 1(b) due to presence of this new quantum phenomenon. Furthermore, the refraction index for purely quantum mode shows an interesting characteristic minimum value properties of which will be studied in terms of different plasma parameters further below. Experimental refractive index of the metallic beryllium and chromium and semiconductor silicon are shown in Fig. 2 [52]. The characteristic minimum similar to that in Fig. 1(c) is clearly apparent which is an indication of the pure quantum collective effect in these metals. Please note that current theory is only supposed to describe the optical properties of simple metals like beryllium and is not capable to contribute to the inter- or intra-band transition effects in semiconductors. This theory however of vital importance in study of optical characteristics of dense hot plasma like compressed beryllium in inertial confined experiments.

In Fig. 3 we have shown the effect of quantum diffraction (indicated by $H$ parameter in calculations) on different optical parameters of dense electron gas with $\eta_{0}=100$ and 


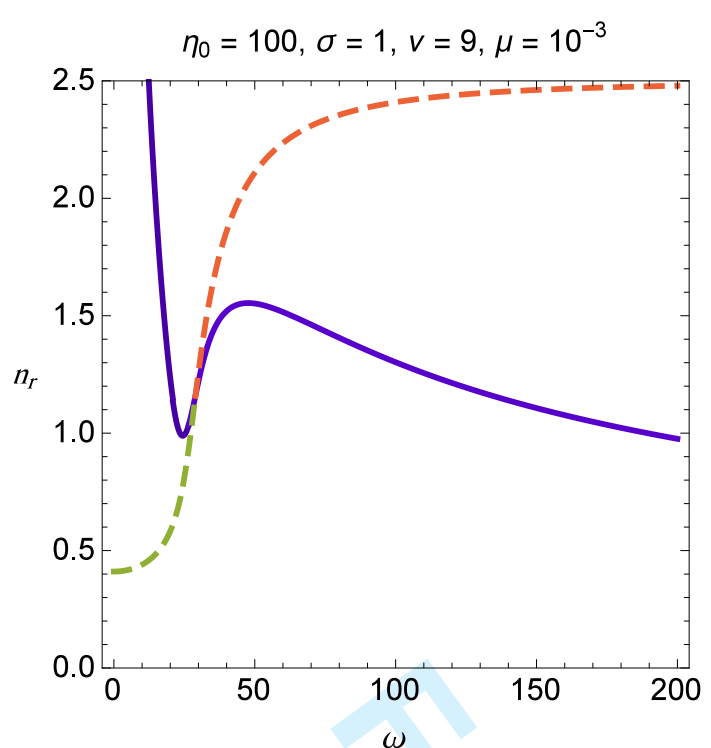

(a)

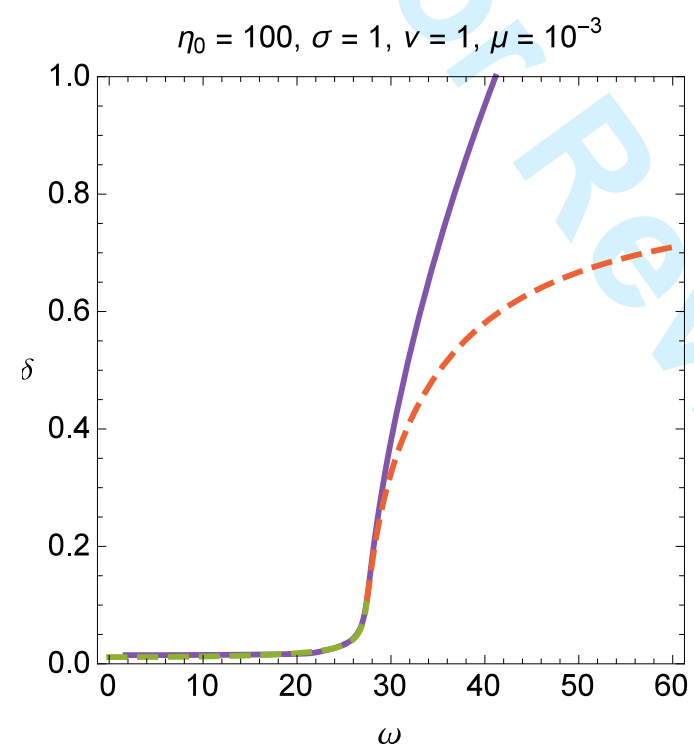

(c)

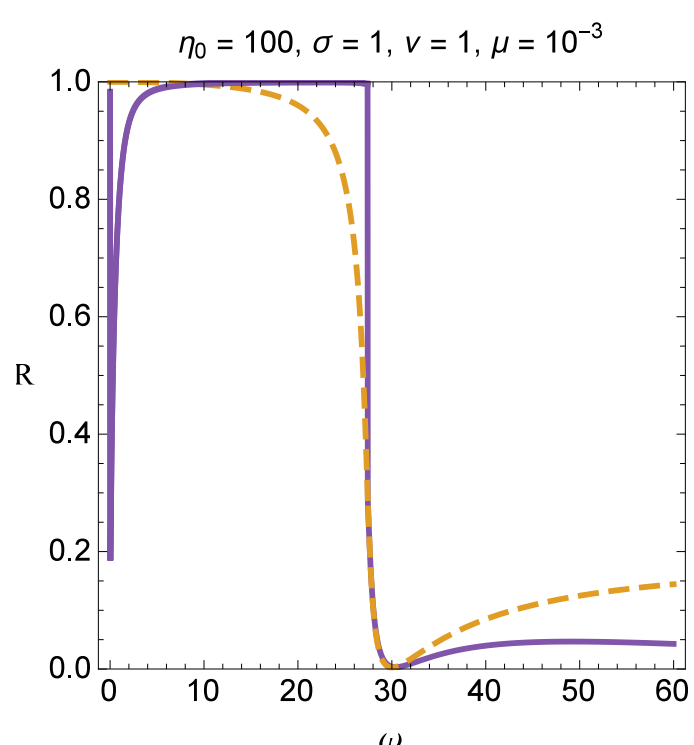

(b)

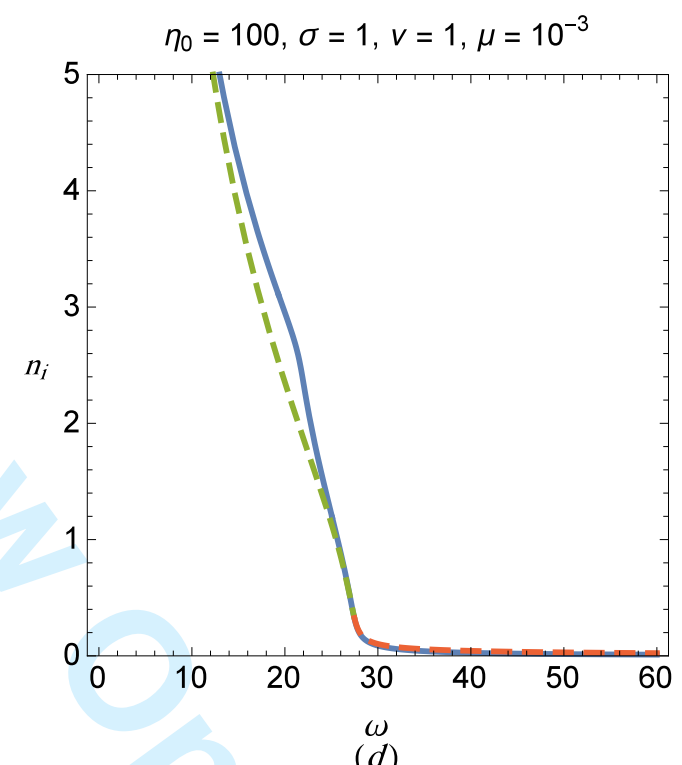

$\stackrel{\omega}{(d)}$

FIG. 3: Different optical parameters of electron gas such as real and imaginary refractive index, reflection coefficient and skin depth for given parameters of the normalized chemical potential $\eta_{0}$, fractional temperature $\sigma=300 K / T_{e}$, collision parameter $\nu$ and magnetic field strength parameter $\mu$. The dashed/solid profile shows the case without/with the collective quantum diffraction effect.

$\sigma=1$ that is $n_{0} \simeq 1.89 \times 10^{22} \mathrm{~cm}^{-3}$ typical of completely degenerate electron Fermi gas in metals. We have only concerned with the pure quantum propagation mode here showing a characteristic minimum in refractive index. Figure 3(a) compares the refractive index with (solid curve) and without (dashed curve) the quantum Bohm potential effect for typical 
plasma parameters of $n_{0} \simeq 1.89 \times 10^{22} \mathrm{~cm}^{-3}, \nu=9$ (high-collision fluid) and $\mu=10^{-3}$ (low magnetic field). The real part of refractive index is evidently resembles that of the experimental one for simple metals in Fig. 2. Moreover, it is observed that the dashed refractive index curve not taking into account the quantum diffraction effect in quite different and can not capture essential features of a quantum electron gas. Figure 3(b) shows the reflection coefficient $R=\left[\left(1-n_{r}\right)^{2}+n_{i}^{2}\right] /\left[\left(1+n_{r}\right)^{2}+n_{i}^{2}\right]$ of the electron fluid with a density typical of metals and low collision rate $(\nu=1)$ and magnetic field $\left(\mu=10^{-3}\right)$. The solid curve contributing to quantum potential effect reveals several important feature. First, a resonance condition is observed to occur at the plasmon frequency $\omega \simeq 30$. Second, a sharp reflection edge (ultraviolet transparency of metals) which is a characteristics of metals [21] is clearly observed. Third, a very low frequency deep minimum is found in the reflection spectrum. From the dashed profile in this figure it is confirmed that the theory ignoring the collective quantum diffraction effect only attributes to the first element, i.e., the resonance feature which is easily described using simple classical Drude-Lorentz model [20]. The third feature in reflection spectrum is specifically more interesting, since, it captures effects beyond that of Drude-Lorentz model which is considered a quite convincing model of free electron gas. In many literature this effect is attributed to the interband transitions (parallel band effect) in metals [20, 21]. For instance, the dip in the reflection spectrum at photon energy of $E_{p h}=1.5 \mathrm{eV}$ in experimental data of Aluminium is attributed to the later effect. It is however quite interesting to see that current theory, which can not attribute to interand intera-band transition effects, successfully predicts a low energy reflectance minimum in quantum electron fluids model for typical metallic electron number-densities.

Figure 3(c) shows the skin depth $\delta=c / \omega n_{i}$ of electron fluid with plasma parameters similar to that in Fig 3(b). For both theory with and without the diffraction effect the skin depth is negligible for frequencies lower that of plasmon excitation which is quite convincing for metals. However, beyond this limit the two theories diverge significantly so that ignoring the quantum potential effect leads to much lower values of skin depth, particularly, for higher frequencies. Figure 3(d) shows the imaginary component of the refractive index and reveals that the predictions of the theory with and without the Bohm correction are only slightly different in this case contrary to the significant difference in the real components. It is however concluded that the quantum Bohm potential is the main ingredient in dynamics of electron fluid in dense plasmas and the study of properties of a dense plasmas without 


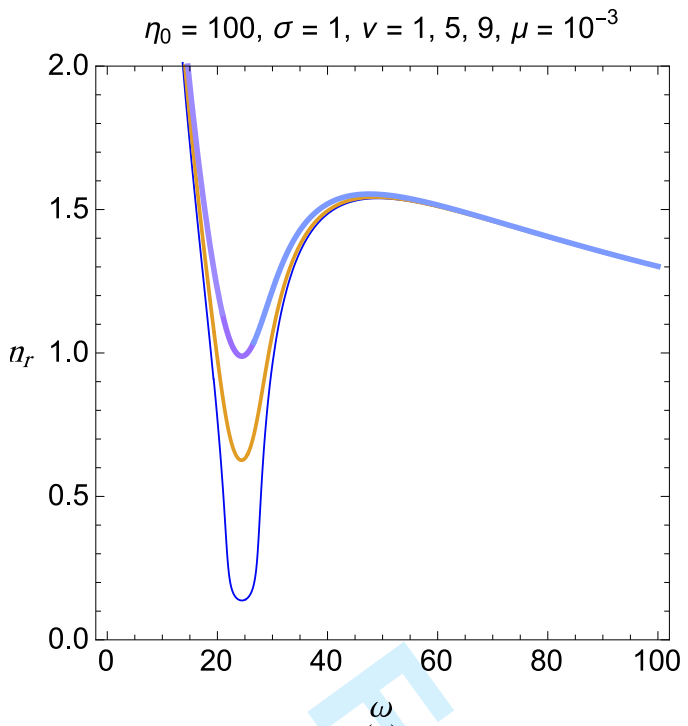

(a)

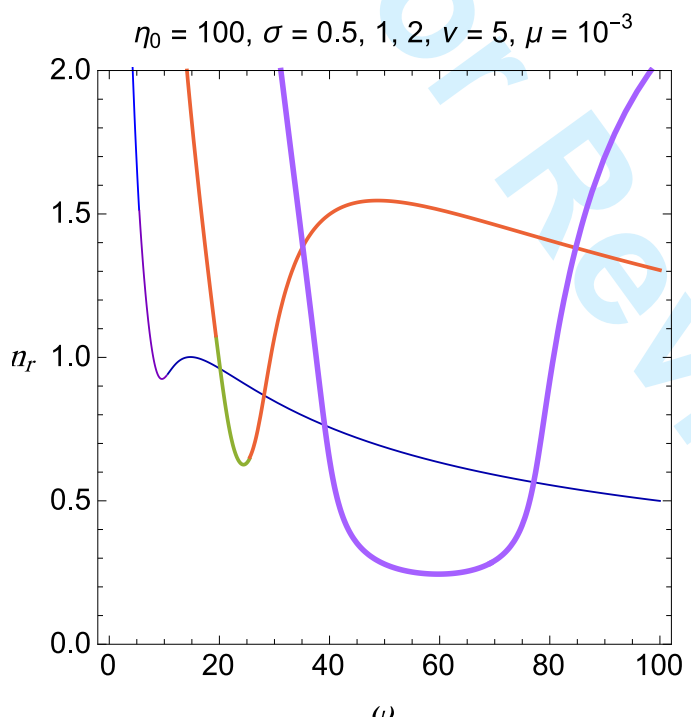

(c)

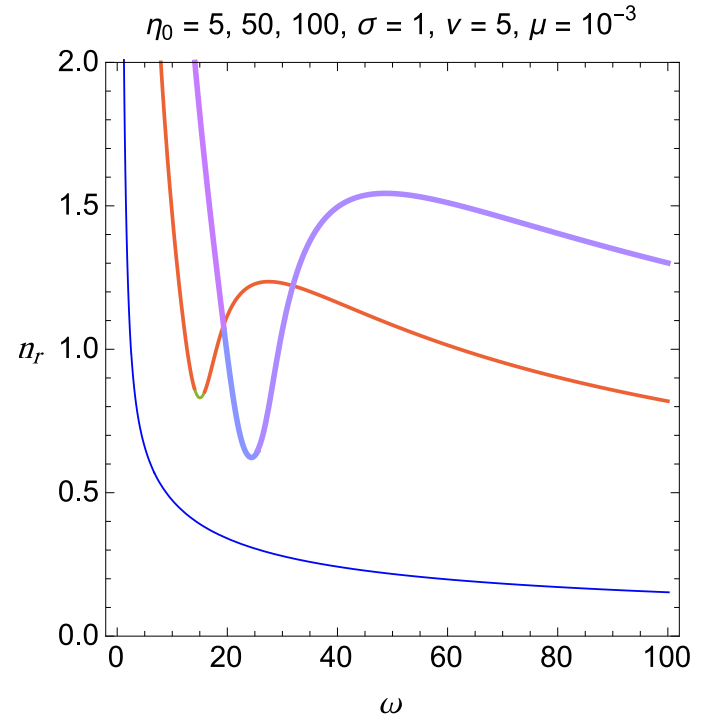

(b)

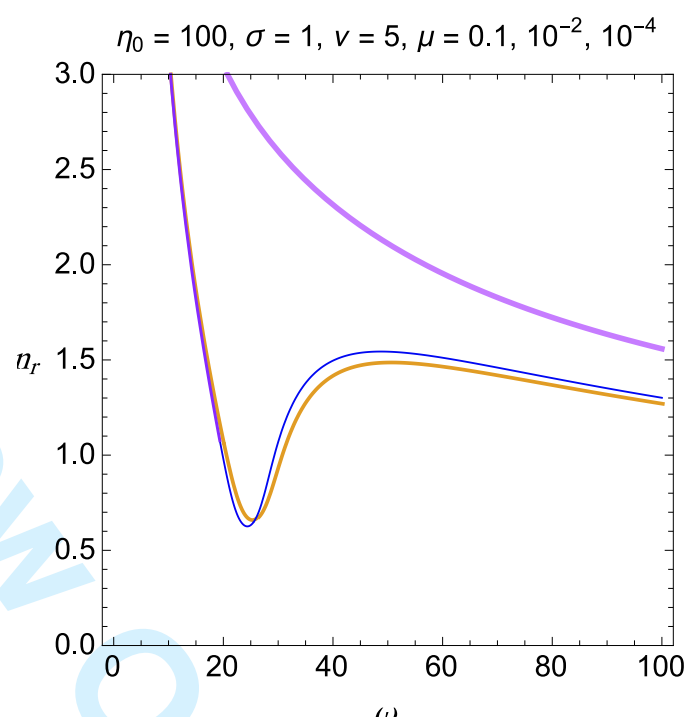

$(d)$

FIG. 4: Dependence of the refraction index of arbitrary degenerate electron fluid on different plasma parameters such as the normalized chemical potential $\eta_{0}$, fractional temperature $\sigma=300 K / T_{e}$, collision parameter $\nu$ and magnetic field strength parameter $\mu$. Increase in the thickness of curves in each plot indicates the increase in the value of varied parameter above each panel.

taking into account this important feature will be incomplete. In figure 4 we have shown the effects of different plasma parameters on the refraction index spectrum and particularly its characteristic minimum. Figure 4(a) reveals that the collision frequency ratio $\nu$ directly alters the depth of the minimum leaving its position unchanged. It is specifically remarked that the increase in the value of $\nu$ causes a sharp decrease in the minimum depth. This 
feature can be quite useful in diagnosing the impurity or defects in metallic crystals, say by annealing process, leading to suppressed electron-phonon or electron-impurity collision rate. Figure 4(b), on the other hand, shows the effect of number-density on the characteristic minimum in refraction index spectrum. It is observed that this minimum is not present for low density fluid, (see for instance the profile for $\eta_{0}=5$ and $\sigma=1$ or $n_{0} \simeq 2.2 \times 10^{20} \mathrm{~cm}^{-3}$ ). It is also observed that compressing the fluid leads to increase in depth and frequency of the minimum. The latter however should be studied considering also the increase in the collision rate due to increase of plasma density, which is has not been taken into account in current investigation. Moreover, Fig. 4(c) indicates that the increase in the value of $\sigma=300 \mathrm{~K} / \mathrm{T}_{e}$ or the decrease in electron fluid temperature leads to increase in both the depth and width of the characteristic minimum. Finally, in Fig. 4(d) the effect of magnetic field strength is investigated on the refraction spectrum indicating that the low magnetic field strength has insignificant effect of the spectrum while the increase beyond a specific threshold leads to complete elimination of the characteristic minimum.

In Fig. 5 we study the influence of different parameters on the reflection spectrum of electron fluid. It is evident fron Fig. 5(a) that increase in the collision frequency leads to an insignificant shift of the reflection edge to lower frequencies. However, the change in the collision parameter $\nu$ significantly modifies the reflection profile very close to the resonance plasmon frequency and low frequency dip in the spectrum. The increase in this parameter is observed to smear out the sharp feature of the resonate condition. Furthermore, Fig. 5 (b) shows that the electron number-density has a profound effect of the reflection profile increase of which moves the reflection edge to higher frequencies. It is also observed that for frequencies higher than the plasmon the reflection spectrum is changed significantly due to variation in the electron density. The decrease in the electron fluid temperature as shown in Fig. 5(c) leads to a significant shift of the reflection edge to higher frequencies as well. Finally, Fig. 5(d) reveals an important feature of the electron fluid. It shows that while the increase in the low strength of magnetic field only slightly alters the reflectance of an electron fluid, it fundamentally changes the spectrum beyond a characteristic high external magnetic field. The later feature is well-known as the magnetic window effect $[53,54]$ and is very useful in elimination of the reentry communication failure.

Figure 6 shows the existence of remarkable low frequency window into the dense quantum electron gas and its parametric dependence on different factors of the fluid. Such a feature 

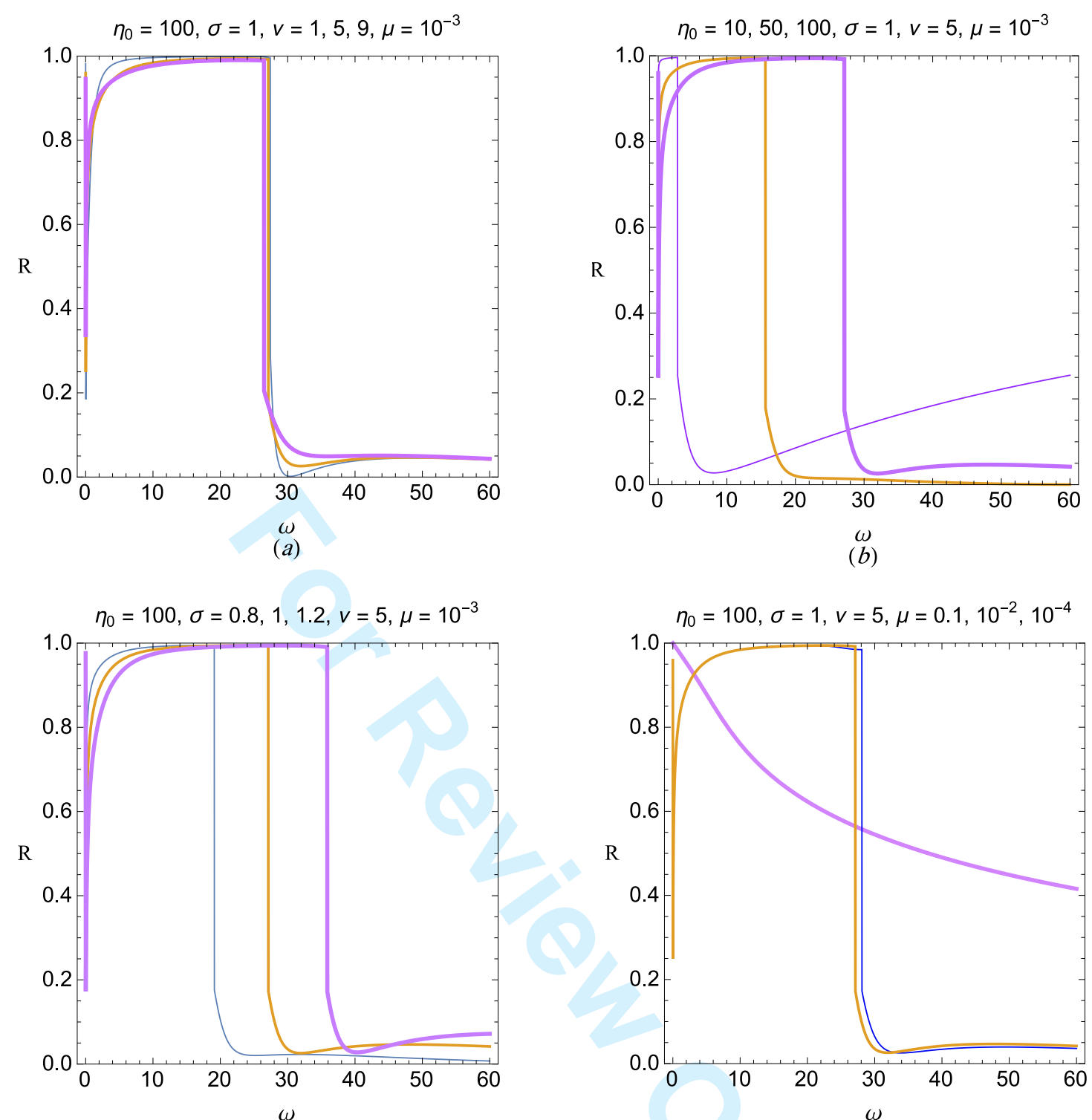

$\stackrel{\omega}{(c)}$

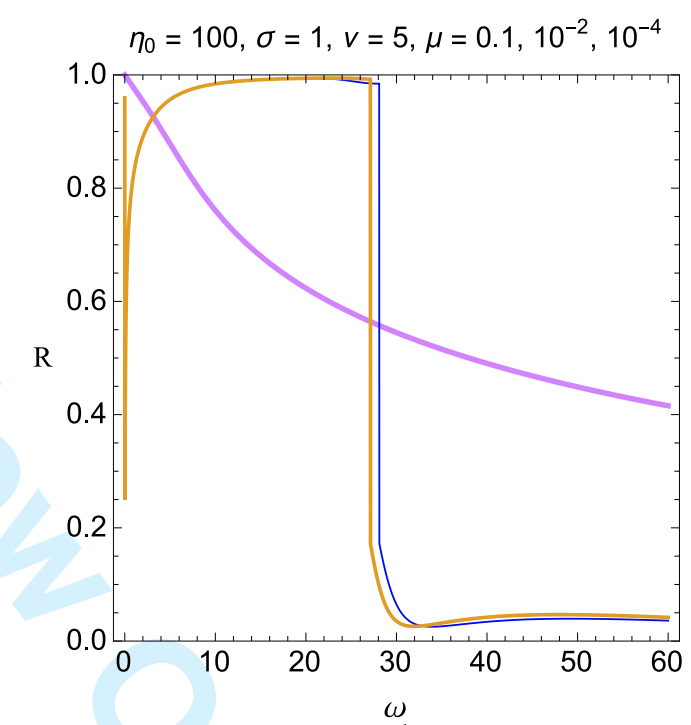

$\stackrel{\omega}{(d)}$

FIG. 5: Dependence of the reflection coefficient of arbitrary degenerate electron fluid on plasma parameters such as the normalized chemical potential $\eta_{0}$, fractional temperature $\sigma=300 K / T_{e}$, collision parameter $\nu$ and magnetic field strength parameter $\mu$. Increase in the thickness of curves in each plot indicates the increase in the value of varied parameter above each panel.

may also be used as a new technic in elimination of reentry communication problem in the absence of an expensive high strength static magnetic field or in study of the dense crust of compact astrophysical objects. It is seen from Fig. 6(a) that the increase in the fluid density increases the width of the low frequency transmission window, the depth of which stays unaffected. Figure 6(b) shows that the decrease of the electron fluid temperature 


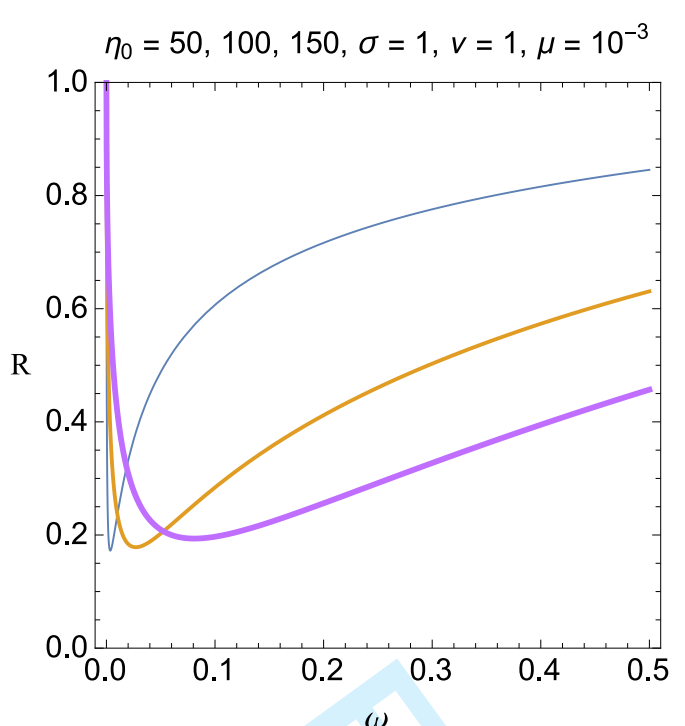

(a)

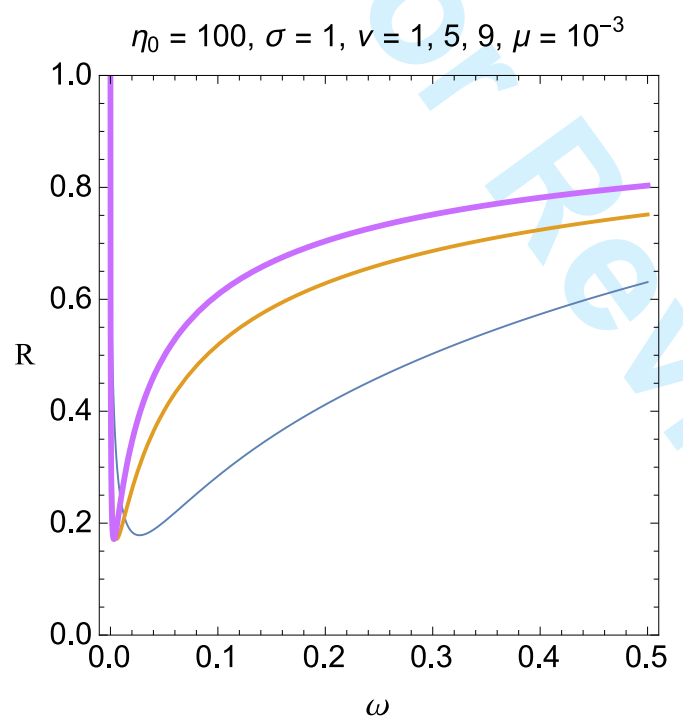

(c)

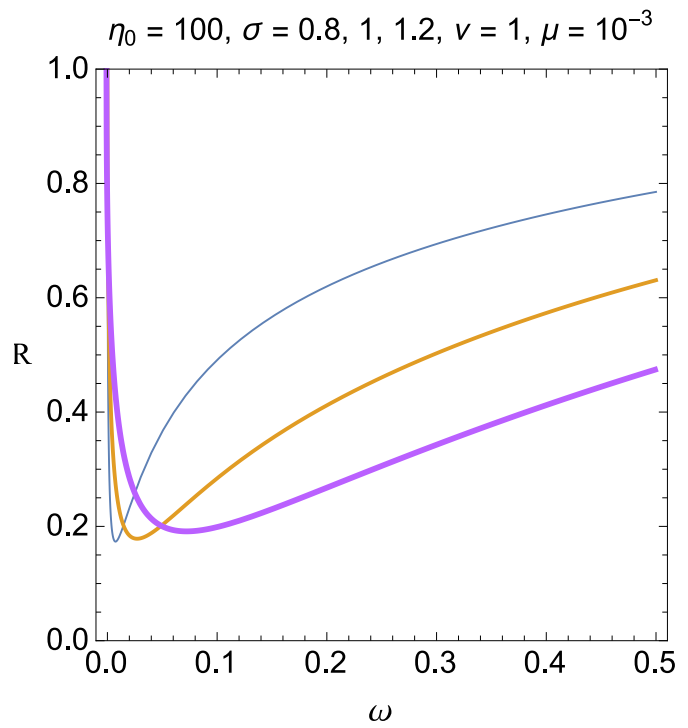

(b)

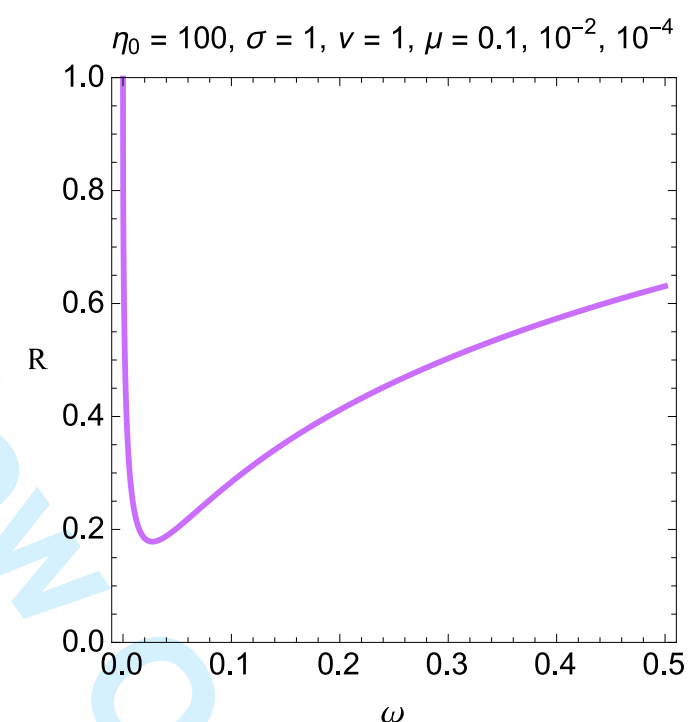

(d)

FIG. 6: Dependence of the width and position of the low energy transmission window on plasma parameters such as the normalized chemical potential $\eta_{0}$, fractional temperature $\sigma=300 K / T_{e}$, collision parameter $\nu$ and magnetic field strength parameter $\mu$. Increase in the thickness of curves in each plot indicates the increase in the value of varied parameter above each panel.

has also similar effect on the width of the transmission window. On the other hand, it is remarked from Fig. 6(c) that the width of the transmission window is also sensitive to the value of the collision frequency parameter, $\nu$. It is observed that the increase in this parameter leads to a sharp decrease in the width. It is also interesting to see from Fig. 6(d) that the magnetic field strength has no effect on the characteristics of this window. 


\section{CONCLUSIONS}

We studied the optical properties of an electron fluid in a quasineutral medium in the framework of appropriate collisional magnetohydrodynamic model which incorporates the quantum statistical and collective quantum diffraction of fluid. The optical parameter such as the complex dielectric response, refraction index, reflection spectrum and skin depth was studied in terms of different factors such as the collision parameter, number density of electrons, magnetic field strength and electron fluid temperature. It was found that this theory can fully describe the essential features of the electron gas from dilute classical up to the fully degenerate quantum regime. A magnetic window was observed to appear by increasing of the transverse magnetic field strength. A sharp reflection edge like that experimentally observed for metals exist for dense electron fluid with any degeneracy characteristics of which was studied in detail. A low frequency window was found to exist which has been previously attributed to interband transition effects in metals. Such a transmission window may be of great value in low frequency communication with the reentry device or the study of optical features of a compact astrophysical object surface through its dense atmosphere.

[1] R. P. Drake, Phys. Plasmas 16, 055501 (2009).

[2] F. Hoyle, F. and W. A. Fowler, Astrophys. J. 132, 565 (1960).

[3] S. Chandrasekhar, "An Introduction to the Study of Stellar Structure", The University of Chicago Press, Chicago (1939).

[4] E. E. Salpeter, ApJ 134669 (1961).

[5] A. Benuzzi-Mounaix, S. Mazevet, A. Ravasio, T. Vinci, A. Denoeud, M. Koenig, N. Amadou, E. Brambrink, F. Festa, A. Levy, et.al., Phys. Scripta T161, 014060 (2014).

[6] Y. Klimontovich and V. P. Silin, in Plasma Physics, edited by J. E. Drummond (McGraw-Hill, New York, 1961).

[7] D. Bohm, Phys. Rev. 85, 166 (1952).

[8] D. Bohm and D. Pines, Phys. Rev. 92, 609 (1953).

[9] D. Bohm and D. Pines, in Plasma Physics, Ed. J. E. Drummond (McGraw-Hill, New York, 1961) Chap. 2, pp. 35-87. 
[10] P. Levine and O. V. Roos, Phys. Rev, 125 207(1962).

[11] D. Pines, Phys. Rev. 92629 (1953).

[12] N. A. Krall \& A. W. Trivelpeice, "Principles of Plasma Physics", (San francisco Press, San francisco 1986).

[13] F. F. Chen, Introduction to Plasma Physics and Controlled Fusion, 2nd ed. (Plenum Press, New York, London, 1984).

[14] N. W. Ashcroft and N. D. Mermin, Solid state physics (Saunders College Publishing, Orlando, 1976).

[15] C. Kittel, Introduction to Solid State Physics, (John Wiely \& Sons, New York, 1996), 7th ed.

[16] G. Manfredi, How to model quantum plasmas, Fields Inst. Commun. 46, 263287 (2005); in Proceedings of the Workshop on Kinetic Theory (The Fields Institute, Toronto, Canada 2004): http://arxiv.org/abs/quant-ph/0505004.

[17] P. K. Shukla and B. Eliasson, Phys. Usp. 51, 53 (2010).

[18] P. K. Shukla and B. Eliasson, Rev. Mod. Phys. 83, 885 (2011).

[19] S. H. Glenzer, O. L. Landen, P. Neumayer, R. W. Lee, K. Widmann, S. W. Pollaine, R. J. Wallace, G. Gregori, A. Holl, T. Bornath, R. Thiele, V. Schwarz, W.-D. Kraeft, R. Redmer, Phys. Rev. Lett. 98, 065002(2007).

[20] F. Wooten, "Optical Properties of Solids", Academic Press, New York and London (1972).

[21] M. Fox, "Optical Properties of Solids", Oxford University Press, New York (2001).

[22] U. Mizutani "”, Cambridge University Press, New York (2001).

[23] N. Crouseilles, P. A. Hervieux, and G. Manfredi, Phys. Rev. B 78, 155412 (2008).

[24] F. Haas, Quantum Plasmas: An Hydrodynamic Approach (Springer, New York, 2011).

[25] A. E. Dubinov, Rus. Microelec. 30, 339(2001).

[26] M. Akbari-Moghanjoughi, Phys. Plasmas 20, 102115(2013).

[27] M. Akbari-Moghanjoughi, Phys. Plasmas, 20, 042706(2013).

[28] M. Akbari-Moghanjoughi, Phys. Plasmas, 20, 092902(2013).

[29] A. Moradi and H. Khosravi, Phys. Rev. B 76, 113411 (2007).

[30] Li Wei and You-Nian Wang, Phys. Rev. B 75, 193407 (2007).

[31] C. L. Gardner, C. Ringhofer, Phys. Rev. E 53, 157 (1996).

[32] C. L. Gardner, SIAM J. Appl. Math. 54, 409 (1994).

[33] M. Akbari-Moghanjoughi and B. Eliasson, Hydrodynamic Theory of Partially Degenerate 
Electron-Hole Fluids in Semiconductors (accepted in Phys. Scripta).

[34] I. Zeba, M. E. Yahia, P. K. Shukla, W. M. Moslem, Phys. Lett. A 376, 2309 (2012).

[35] F. Haas, G. Manfredi and M. Feix, Phys. Rev. E 62, 2763 (2000).

[36] F. Haas, G. Manfredi and J. Goedert, Braz. J. Phys. 33, 128 (2003).

[37] Fernando Haas and Bengt Eliasson, Phys. Scr. 90, 088005 (2015).

[38] B. Eliasson and P. K. Shukla, Phys. Scr. 78, 025503 (2008).

[39] F. Haas and S. Mahmood, Phys. Rev. E 92, 053112 (2015).

[40] A. Bret, Phys. plasmas 14, 084503 (2007).

[41] A. Bret, Phys. plasmas 15, 022109 (2008).

[42] P. K. Shukla and B. Eliasson, Phys. Rev. Lett. 108, 165007 (2012); 108, 219902 (E) (2012); ibid. 109, 019901 (E) (2012).

[43] B. Eliasson, M. Akbari-Moghanjoughi, Phys. Lett. A 380, 2518 (2016).

[44] M. Marklund and P. K. Shukla, Rev. Mod. Phys. 78, 591 (2006).

[45] G. Brodin, M. Marklund, and G. Manfredi, Phys. Rev. Lett. 100, 175001 (2008).

[46] M. Marklund, G. Brodin, L. Stenflo, and C. S. Liu, Europhys. Lett. 84, 17006 (2008).

[47] G. Brodin and M. Marklund, New J. Phys. 9, 277 (2007).

[48] M. Akbari-Moghanjoughi, Phys. Plasmas 17, 137709(2010).

[49] M. Akbari-Moghanjoughi, Phys. Plasmas 18, 012701(2011).

[50] M. Akbari-Moghanjoughi, Phys. Plasmas 21, 062103 (2014).

[51] P. K. Shukla, Phys. Lett. A 369 312(2007).

[52] M. N. Polyanskiy. "Refractive index database," http://refractiveindex.info

[53] A. Mousavi, A. Esfandiari-Kalejahi, and M. Akbari-Moghanjoughi, Phys. Plasmas 23, 043516 (2016).

[54] A. Mousavi, A. Esfandiari-Kalejahi, and M. Akbari-Moghanjoughi, Phys. Plasmas 23, 073511 (2016). 